DOI: https://doi.org/10.34069/RA/2021.7.02

Volumen 4, Número 7/enero-junio 2021

\title{
Aporte de la vegetación existente en agroecosistemas suburbanos de Santiago de Cuba a la alimentación
}

\author{
Contribution of existing vegetation in suburban agroecosystems of Santiago de Cuba to \\ food
}

\section{Contribuição da vegetação existente nos agroecossistemas suburbanos de Santiago de Cuba para a alimentação}

Recibido: 2 de febrero de $2021 \quad$ Aceptado: 23 de abril de 2021

\author{
Autores: \\ Rubert Rodríguez Fonseca ${ }^{6}$ \\ Wilder Garcés Castillo ${ }^{7}$ \\ Belyani Vargas Batis ${ }^{8}$ \\ Randy González Amita ${ }^{9}$
}

\section{Resumen}

En los momentos actuales alcanzar la seguridad alimentaria resulta vital, por tanto, el análisis de cómo contribuyen los diferentes tipos de sistemas productivos al logro este objetivo es de suma importancia. El trabajo se centró en valorar la percepción de los productores y consumidores sobre el aporte de la vegetación dedicada a la producción de alimentos, en agroecosistemas suburbanos de Santiago de Cuba, a la alimentación. Seleccionadas las fincas se realizaron muestreos para establecer un listado base sobre las especies de plantas encontradas en las fincas, luego se aplicaron entrevistas semiestructuradas a productores y consumidores en las localidades cercanas a cada uno de los sistemas agrícolas. En el listado elaborado se contabilizaron 185176 individuos, 33 familias, 55 géneros y 72 especies. La composición de plantas destinadas para la alimentación es bastante estable y las especies sobre las cuales se sustenta la dieta de productores y consumidores tienen una representación superior al $59 \%$ destacándose los vegetales, viandas y frutas frescas. Hay correspondencia entre las formas de adquirir los productos por parte de los consumidores y las formas de comercialización utilizadas por los productores. Las hortalizas, viandas y frutas frescas son los productos más adquiridos por los consumidores y a los que más acceso tienen los productores con un reconocimiento de la disponibilidad y la calidad superior al $50 \%$. La flora cultivada con fines alimenticios en las fincas suburbanas estudiadas contribuye al logro de la seguridad alimentaria.

Palabras clave: Alimentación, hortalizas, frutas, disponibilidad, calidad.

\begin{abstract}
At present, achieving food security is vital; therefore, the analysis of how the different types of production systems contribute to achieving this objective is of utmost importance. The work focused on assessing the perception of producers and consumers about the contribution of the vegetation dedicated to food production, in suburban agroecosystems of Santiago de Cuba, to

\footnotetext{
${ }^{6}$ Departamento de Agronomía. Universidad de Oriente. Santiago de Cuba. Cuba. https://orcid.org/ 0000-0002-6032-6438

${ }^{7}$ Departamento de Agronomía. Universidad de Oriente. Santiago de Cuba. Cuba. https://orcid.org/0000-0003-2068-1408

${ }^{8}$ Departamento de Agronomía. Universidad de Oriente. Santiago de Cuba. Cuba. https://orcid.org/0000-0002-6698-1281

${ }^{9}$ Empresa de Cultivos Varios "Laguna Blanca". Contramaestre. Santiago de Cuba. Cuba. https://orcid.org/0000-0002-0920-6342
} 
food. Once the farms were selected, samplings were carried out to establish a base list of the plant species found on the farms, and then semi-structured interviews were applied to producers and consumers in the localities close to each of the agricultural systems. 185176 individuals, 33 families, 55 genera and 72 species were counted in the list prepared. The composition of plants intended for food is quite stable and the species on which the diet of producers and consumers is based have a representation of more than 59\%, with vegetables, meats and fresh fruits standing out. There is a correspondence between the ways consumers purchase products and the marketing ways used by producers. Vegetables, meats and fresh fruits are the products most purchased by consumers and to which producers have the most access, with recognition of availability and quality of more than 50\%. The flora cultivated for food purposes on the studied suburban farms contributes to the achievement of food security.

Keywords: Food, vegetables, fruits, availability, quality.

\section{Resumo}

Actualmente, o alcance da segurança alimentar é vital, pelo que a análise de como os diferentes tipos de sistemas de produção contribuem para a concretização deste objectivo é da maior importância. O trabalho se concentrou em avaliar a percepção de produtores e consumidores sobre a contribuição da vegetação dedicada à produção de alimentos, em agroecossistemas suburbanos de Santiago de Cuba, para a alimentação. Uma vez selecionadas as fazendas, foram realizadas amostragens para estabelecer uma lista de base das espécies vegetais encontradas nas fazendas, em seguida, foram aplicadas entrevistas semiestruturadas com produtores e consumidores das localidades próximas a cada um dos sistemas agrícolas. 185176 indivíduos, 33 famílias, 55 gêneros e 72 espécies foram contados na lista elaborada. A composição das plantas destinadas à alimentação é bastante estável e as espécies que servem de base à dieta alimentar dos produtores e consumidores têm uma representação superior a 59\%, com destaque para os vegetais, carnes e frutas frescas. Existe uma correspondência entre as formas como os consumidores compram os produtos e as formas de comercialização utilizadas pelos produtores. Legumes, carnes e frutas frescas são os produtos mais comprados pelos consumidores e aos quais os produtores têm mais acesso, com um reconhecimento de disponibilidade e qualidade de mais de 50\%. A flora cultivada para fins alimentares nas fazendas suburbanas estudadas contribui para o alcance da segurança alimentar.

Palavras-chave: Alimentos, vegetais, frutas, disponibilidade, qualidade.

\section{Introducción}

La agricultura a pequeña escala (APE) hace referencia a propiedades agrícolas en las que la mayor parte de las iniciativas provienen de la familia, la que invierte gran parte de su tiempo de trabajo en dicha propiedad. Agrupa a pequeños agricultores con una baja base de activos que manejan menos de 2 ha de tierra y que practican una mezcla de producción comercial y de subsistencia o cualquiera de ellas. Si bien existe el consenso de que las pequeñas propiedades agrícolas se manejan a nivel familiar y utilizan su mano de obra (Proctor y Lucchesi, 2012), también se refiere que es la principal fuente de alimentación de más del $70 \%$ de la población mundial (Vázquez, 2017).

La producción de alimentos dentro del perímetro urbano, como forma de APE, tiene en cuenta la interrelación seres humanos-cultivo-animal-medio ambiente, así como, la estabilidad de la fuerza de trabajo y la producción diversificada de cultivos y animales. De acuerdo con Páez (2013) la concentración de altas poblaciones genera una alta demanda de productos alimenticios (hortalizas, frutas frescas, flores, condimentos y crianza intensiva de animales). Sin embargo, la reducción de la calidad del producto y el encarecimiento de los costos de producción derivados 
de la transportación a grandes distancias, ha constituido una de las principales premisas para el desarrollo de la producción de alimentos lo más cerca posible del consumidor. Todos estos factores contribuyeron al surgimiento del Programa Nacional de la Agricultura Urbana (AU), Suburbana (ASU) y Familiar (AF) en Cuba.

Este programa ha sido un contribuidor por excelencia de la canasta básica con un alto compromiso a nivel local. Desde sus inicios se articuló con la red de productores en el perímetro urbano de ciudades y pueblos, fomentando la producción vegetal y animal a pequeña escala. Otro de sus aportes significativos es la generación de nuevas fuentes de empleo a mujeres y hombres próximos a sus hogares. Por otra parte, esta forma de producción demanda grandes volúmenes de semillas para mantener una producción estable durante todo el año, la posibilidad de explotar eficientemente el área productiva, así como, mantener e incrementar una amplia diversidad vegetal (DV) pues es la base para la producción de alimentos (Ministerio de la Agricultura [MINAG], 2015).

La DV es esencial para la agricultura, la cual, desarrollada de manera sostenible produce beneficios socioeconómicos duraderos. La conservación sostenible de la biodiversidad (BDV) es importante, la agricultura y la humanidad dependen de ella (Secretaría del Convenio sobre la Diversidad Biológica (CDB), 2008). Los policultivos son una forma de manejar la DV, con su aplicación puede aumentar la producción y diversidad de alimentos, incrementar los ingresos de los productores, mejorar el uso de la tierra e incrementar la ganancia total en el rendimiento del sistema productivo. Tanto la conservación de la BDV para la alimentación y la agricultura como su uso sostenible, son necesarios para proporcionar alimentos, mejorar la situación económica, social y medioambiental de las personas, así como, satisfacer las necesidades de las generaciones futuras Organización de la Naciones Unidas para las Alimentación y la Agricultura (FAO, 2015).

El consumo de nutrientes esenciales y de otros elementos importantes depende de la variedad en los tipos de alimentos y dentro de cada tipo de alimentos que se consume. La BDV es la base de la cadena alimentaria y su uso es importante para la seguridad alimentaria (SA) y nutricional como mecanismo de defensa contra el hambre, fuente de nutrientes para una dieta diversa y de calidad e ingrediente básico para fortalecer los sistemas de alimentación locales y la sostenibilidad del medioambiente. Mediante la BDV, la agricultura proporciona una amplia gama de energía, proteínas, grasas, minerales, vitaminas y otros micronutrientes claves para la SA y la nutrición (Rah et al., 2010).

De acuerdo con el Consejo Nacional de Evaluación de la Política de Desarrollo Social [CONEVAL] (2010) existe SA a nivel de individuo, hogar, nación y global, cuando todas las personas, en todo momento, tienen acceso físico y económico a suficiente alimento, seguro y nutritivo, para satisfacer sus necesidades alimenticias y sus preferencias, con el objeto de llevar una vida activa y sana. Según la FAO (2011a), el mundo produce suficiente cantidad de alimentos para satisfacer la demanda de toda la población, la causa básica del hambre y la desnutrición no es la falta de alimentos sino la falta de acceso a los alimentos disponibles. De ahí que la SA requiere que los alimentos estén disponibles todo el tiempo lo que significa que tanto la producción como el acceso deben ser estables.

En agroecosistemas suburbanos de Santiago de Cuba los estudios florísticos que se realizan son muy parcializados en los que se evalúan solo aspectos relacionados con la complejidad del sistema o el comportamiento sinecológico de las especies. De la literatura consultada solo los estudios realizados por Vargas et al. (2016), Vargas et al. (2017) y Vargas et al. (2019) hacen de alguna manera referencia a grupos vegetales objeto de cultivo, pero sin analizar su implicación en la SA. Se precisa entonces de investigaciones que realicen aportes al conocimiento en este campo de acción, que sirvan de base para acercar la producción a lo que quiera, necesite y pueda adquirir el consumidor. 


\section{AMAZÓNAS}

\section{Objetivo general}

Valorar la percepción de los productores y consumidores sobre el aporte de la vegetación dedicada a la producción de alimentos, en agroecosistemas suburbanos de Santiago de Cuba, a la alimentación.

\section{Marco teórico}

La APE es una modalidad de producción que se desarrolla en fincas con una extensión por debajo de 2 ha. Sus propietarios deben tener acceso a la tierra, el agua y las semillas, así como, a los recursos productivos y servicios públicos adecuados. La misma contribuye de forma importante a los servicios ambientales. Es practicada por familias, en las que figuran uno o más hogares, que utilizan solo o mayormente, mano de obra familiar y que obtienen de ese trabajo una parte considerable, pero variable, de sus ingresos, en especie o en dinero. Comprende el cultivo, la cría de animales, la actividad forestal y la pesca artesanal (Lipper y Neves, 2011). La finalidad principal es mejorar las condiciones de vida de los productores y promover sus productos. En el mundo existen diferentes programas que se encargan de incentivar la APE dentro de los cuales se señala el fomento de la AU (Velasco, 2014).

En Cuba la APE se desarrolla a partir del ya mencionado Programa Nacional de AU, ASU y AF. Los huertos intensivos, organopónicos, patios familiares, así como, los cultivos protegidos y semiprotegidos son algunas de las modalidades referidas por Vargas (2006) para este programa. Las fincas suburbanas se añadieron a estas modalidades, según Orellana (2010), desde que la FAO en 1999 adicionó a la denominación del programa el término de Periurbana o Suburbana.

Los pequeños productores contribuyen a la SA y la nutrición a escala mundial, al tiempo que desempeñan otras funciones conexas en sus territorios. Los datos históricos indican que la APE, debidamente respaldada por las políticas e inversiones públicas, tiene la capacidad de contribuir de manera efectiva a la SA. Aporta al crecimiento económico, la generación de empleo, la reducción de la pobreza, la emancipación de grupos desatendidos y marginados y la reducción de las desigualdades espaciales y socioeconómicas. En un entorno político e institucional propicio, puede contribuir a la conservación del patrimonio cultural y a la ordenación sostenible de la BDV y otros recursos naturales (FAO, 2010). De lo planteado se entiende que la APE realiza importantes contribuciones al logro de la SA. El High Level Panel of Experts [HLPE] (2013) señaló que al analizar esta contribución debe hacerse en relación con sus cuatro dimensiones.

A partir de la proposición de que la SA, en última instancia, busca el bienestar nutricional y tomando en cuenta que esta forma parte del desarrollo humano sostenible, se pueden obtener varias definiciones. Pero, indistintamente de las definiciones, según Paz (2003), quedan como elementos sustentadores del concepto los siguientes aspectos. De acuerdo con el Instituto de Nutrición de Centro América y Panamá [INCAP] (2004) SA y nutricional es el estado en el cual todas las personas gozan en forma oportuna y permanente de acceso a los alimentos que necesitan, en cantidad y calidad para su adecuado consumo y utilización biológica garantizándole un estado de bienestar que coadyuve al desarrollo humano.

Guardiola et al. (2006), señalaron que la SA es un estado en el que todas las personas en todo momento tienen acceso físico y económico a suficiente alimento para satisfacer sus necesidades dietéticas para una vida productiva y saludable. La SA es un fenómeno destacado en el área rural, debido a la importancia que tiene esta área en cuanto a la producción de alimentos y su vulnerabilidad a las inclemencias del tiempo, así como, en la falta de acceso a los mismos. Según la FAO (2011b) la SA existe cuando todas las personas tienen, acceso físico, social y económico a alimentos suficientes, inocuos y nutritivos que satisfacen sus necesidades energéticas diarias para llevar una vida activa y sana. De acuerdo con Castro (2013) la SA, para 
todos, será posible si adquiere la condición de bien público mundial y de objetivo central tanto para los gobiernos como para el desarrollo nacional.

Existen diferentes dimensiones para medir la SA; se describen las más usadas. Debe señalarse que cada dimensión es condición necesaria, pero no suficiente para la siguiente (CONEVAL, 2010). A medida que se ha estudiado y profundizado en el tema, el concepto de seguridad ha cambiado en el tiempo. Inicialmente solo se consideraba la disponibilidad de alimentos, actualmente se tiene la percepción de que existen otros elementos igualmente importantes de ahí que, la SA conste, según Ardón (2012), de cuatro dimensiones.

Disponibilidad de alimentos: Es la primera etapa de la SA. La disponibilidad de alimentos es resultado de la producción interna, del nivel de las reservas, las importaciones y exportaciones, las ayudas alimentarias y la capacidad de almacenamiento y movilización. Esta disponibilidad debe ser estable de forma que existan alimentos suficientes durante todo el año. También debe ser adecuada a las condiciones sociales y culturales, y con productos inocuos, es decir, sin sustancias dañinas para la salud (CONEVAL, 2010).

Acceso a los alimentos: Una oferta adecuada de alimentos a nivel nacional o internacional en sí no garantiza la SA a nivel de los hogares. La preocupación acerca de una insuficiencia en el acceso a los alimentos ha conducido al diseño de políticas con mayor enfoque en materia de ingresos y gastos, para alcanzar los objetivos de SA (FAO, 2011b).

Consumo y utilización de los alimentos: Esta dimensión se refiere al conocimiento que tiene la población sobre los alimentos (como prepararlos, componentes nutricionales) y a la capacidad de asimilar los nutrientes de los alimentos que consumen (Ardón, 2012).

Estabilidad en el tiempo: En el caso de que la ingesta de alimentos sea adecuada en la actualidad, se considera que no gozan de completa SA si no tienen asegurado el debido acceso a los alimentos de manera periódica, porque la falta de tal acceso representa un riesgo para la condición nutricional (FAO, 2011b).

Ardón (2012) refirió que en el estudio y análisis de la SA es necesario considerar otros aspectos. En primer lugar la cantidad, que tiene relación con los requerimientos mínimos de nutrientes que la persona necesita ingerir para tener una vida saludable. Como segundo aspecto está la calidad, que significa acceso a los alimentos que contengan la mayor cantidad de nutrientes posibles. En tercer lugar, es necesario considerar la regularidad asociada a la continuidad en la ingesta de alimentos por una persona durante un día. Finalmente, la dignidad debido a que, una persona que se alimenta de los desperdicios de los alimentos aunque no esté en condiciones de subnutrición se considera como parte del grupo que vive en inseguridad alimentaria.

\section{Metodología}

La investigación se llevó a cabo en el Departamento de Agronomía de la Universidad de Oriente y en fincas de la ASU del municipio Santiago de Cuba perteneciente a la provincia homónima en el período comprendido de enero de 2017 hasta diciembre de 2020. En dicha época se enmarcan las dos etapas que se consideran en el desarrollo de la agricultura en Cuba (período lluvioso y poco lluvioso). El procedimiento empleado en cada fase de trabajo se describe a continuación.

El trabajo se llevó a cabo en las 10 fincas suburbanas seleccionadas por Guerrero (2017) y Valdés (2017). Los nombres y coordenadas geográficas de las mismas se muestran a continuación: Erick Vega (Latitud norte: 20.091236 y Longitud oeste: 75.786977), La Esperanza (Latitud norte: 20.047084 y Longitud oeste: 75.791690), Tres Palmas (Latitud norte: 20.064135 


\section{AMAZÓNAS}

y Longitud oeste: 75.801778), La República (Latitud norte: 20.068167 y Longitud oeste: 75.801893), La Caballería (Latitud norte: 20.047843 y Longitud oeste: 75.794819), Los Cascabeles (Latitud norte: 20.057827 y Longitud oeste: 75.800777), La Juliana (Latitud norte: 20.086979 y Longitud oeste: 75.793355), El Sol (Latitud norte: 20.090400 y Longitud oeste: 75.800728), La Sorpresa (Latitud norte: 20.038776 y Longitud oeste: 75.789878 ) y La Cecilia (Latitud norte: 20.089008 y Longitud oeste: 75.785528).

Primeramente se estableció un listado base de las plantas sobre la cuales se sustenta la producción de alimentos incluyendo aquellas que, sin ser alimenticias, tienen importancia desde el punto de vista económico. En este sentido las plantas fueron organizadas en dos grupos: $(i)$ plantas destinadas para la alimentación (PDPA) y (ii) especies frutales (EF). Se realizaron recorridos en cada una de las fincas para el muestreo de las especies de cada grupo estableciéndose parcelas con dimensiones de $100 \mathrm{~m}^{2}(10 \mathrm{~m} \times 10 \mathrm{~m})$. El número de parcelas fue variable en dependencia del área total de cada finca y en los dos períodos evaluados fueron utilizadas las mismas parcelas. Siempre se cuidó que el área muestreada fuera al menos el $70 \%$ del total.

La información obtenida en los muestreos de cada una de las especies (nombre vulgar y número de individuos) en las parcelas levantadas fue recogida en hojas de campo datos que fueron utilizados durante la identificación. La identificación se realizó en el Departamento de Agronomía apoyado en la obra de Roig (1988). Los taxas cuya identificación resultó compleja por la vía antes descrita, fueron clasificados por los especialistas del Herbario de BIOECO (Centro Oriental de Ecosistemas y Biodiversidad). Luego de la identificación, los nombres científicos obtenidos fueron actualizados según Acevedo y Strong (2012) y el inventario preliminar de las Plantas Vasculares de Cuba de Greuter y Rankin (2017).

Obtenido el listado base se procedió a la valoración de la percepción de los productores y consumidores sobre como aporta la vegetación dedicada a la producción de alimentos en estas fincas, al logro de la SA. En el caso de la percepción que poseen los productores sobre las especies de plantas que se destinan para la alimentación en las fincas estudiadas, se aplicó una entrevista semiestructurada en cada sistema productivo. Es válido destacar que aunque todas las personas relacionadas con el sistema agrícola podían participar de la entrevista no todos estuvieron incluidos en la muestra. La muestra estuvo compuesta por el productor propietario y la esposa del productor propietario. En total dos participantes por cada finca lo cual implica una suma de 20 informantes clave si se consideran todas las fincas implicadas en el estudio (10). En este caso se establecieron los siguientes indicadores de evaluación: $(i)$ listado de productos y porcentaje de referencia de los alimentos que son consumidos y que no son producidos en las fincas y (ii) porcentaje de referencia de las diferentes formas de comercialización de productos agrícolas utilizados.

En el caso de la percepción de los consumidores también se realizó una entrevista semiestructurada, esta vez a los habitantes de las comunidades cercanas a las fincas objeto de estudio. Para ello las localidades cercanas a las fincas fueron agrupadas en las cuatro áreas geográficas siguientes según las referidas por del Toro et al. (2018), Vargas et al. (2019b), del Toro et al. (2019) y Vargas et al. (2020): (i) localidades comprendidas entre la carretera a La República-Entronque Caney (incluye las fincas La Caballería, La Esperanza, La Sorpresa), (ii) localidades comprendidas entre la Autopista Nacional-Poblado La República (incluye las fincas Los Cascabeles, Tres Palmas, La República), (iii) localidades comprendidas entre la Autopista Nacional-Poblado de Boniato (incluye las fincas La Juliana, El Sol) y (iv) localidades comprendida entre la Autopista Nacional-Carretera al Cristo y San Vicente (incluye las fincas Erick Vega, La Cecilia).

En cada una de las áreas se aplicó una entrevista a 10 personas para una muestra final de 40 informantes clave seleccionados según el método de Bola de nieve de acuerdo con Vargas et 


\section{AMAZÓNAS}

al. (2011). Aplicada la entrevista, las respuestas fueron agrupadas según consenso entre los informantes (CI) considerando el número de ellos que citó un determinado producto y las cuestiones relacionadas con el, respecto al total de informantes $(\mathrm{N}=40)$ x 100. Obtenida la información para el caso específico de los consumidores, se establecieron los siguientes indicadores de evaluación: (i) vías de adquisición de los productos alimenticios y porcentaje de referencia para cada una de las vías referidas y (ii) frecuencia de compra de los productos y porcentaje de referencia según frecuencia de compras.

Para ambos grupos de informantes clave se evaluó: (i) listados de productos obtenidos de plantas que utilizan para su alimentación, (ii) porcentaje de coincidencia de los productos referidos con los identificados en las fincas, (iii) porcentaje de referencia de cada uno de los productos que son consumidos, (iv) criterios que sustentan la importancia de consumir productos obtenidos a partir de fincas y el porcentaje de referencia para cada criterio, $(v)$ opciones de disponibilidad de los productos que son consumidos y porcentaje de referencia según opciones de disponibilidad y ( $v i$ ) clasificación de la calidad de los productos que son adquiridos y porcentaje de referencia según clasificación de la calidad.

\section{Resultados}

Durante la investigación, se elaboró un listado que incluía 185176 individuos de 33 familias, 55 géneros y 72 especies. Para las PDPA (período poco lluvioso) se reportaron 120596 individuos, 18 familias, 33 géneros y 42 especies en tanto para el período lluvioso se encontraron 61193 individuos, 18 familias, 32 géneros y 40 especies. Por su parte en el grupo de las EF se contaron en la etapa poco lluviosa un total de 1601 individuos, 19 familias, 23 géneros y 27 especies, mientras que en la temporada lluviosa se reportaron 1786 individuos, 17 familias, 20 géneros y 26 especies.

Los productores refirieron un total de 47 especies que utilizan en la alimentación, de ellas 46 se encontraron en el período poco lluvioso y 40 en el lluvioso, lo que representa 66,67 \% y $60,61 \%$ de las que aparecen en cada una de las temporadas respectivamente. En el caso de los consumidores fueron referidas 52 especies de plantas como componentes de sus dietas frecuentes de consumo. De estas especies, 44 pertenecen a la temporada poco lluviosa que representan el $63,77 \%$ del total que se encontraron en el período y 39 son de la época lluviosa y representan el59, $09 \%$ del total para esta temporada.

\begin{tabular}{|c|c|}
\hline - Especie de planta & Porcentaje \\
\hline Puesco macto Kasa & $100 \mathrm{~s}$ \\
\hline 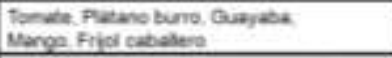 & 905 \\
\hline $\begin{array}{l}\text { Calabara Flatino tida Aguacabe, Finta } \\
\text { boriba Limon. }\end{array}$ & $90 \leqslant$ \\
\hline Fivd gundu Blonivis Coese & $70 s$ \\
\hline Qimbonbe & $65 \$$ \\
\hline Mezz. Berieniztru & $\cos$ \\
\hline Ouenabene Lectugs. Pinients & 505 \\
\hline Hotichuele Zonohure & 405 \\
\hline 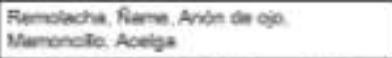 & $30 \leqslant$ \\
\hline 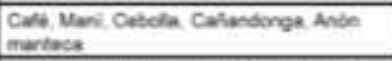 & 205 \\
\hline 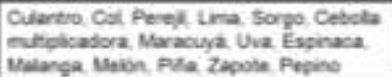 & tos \\
\hline
\end{tabular}

Figura 1. Porcentaje de referencia de las plantas más utilizadas por los productores en su alimentación. 


\section{AMAZđ̛́nAS}

\begin{tabular}{|c|c|}
\hline Especie de planta & Porcentaje \\
\hline Avroz & 81.900 \\
\hline Chichero & $71-908$ \\
\hline Frial Yuca Malanga & $51-60 \%$ \\
\hline $\begin{array}{l}\text { Tomate, Beriato, Calabaza. Maroo, } \\
\text { Gugyaba, Cebolu, Maiz }\end{array}$ & $41.50 \%$ \\
\hline Platane burro, Platano macho, Quimbombo & $31.40 \%$ \\
\hline $\begin{array}{l}\text { Pepino, Col, Pimiento, Ajo, Chapo, Coco, } \\
\text { Zapote }\end{array}$ & $21-30 \%$ \\
\hline 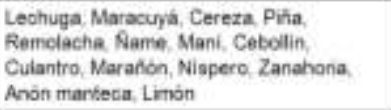 & $11-20 \%$ \\
\hline 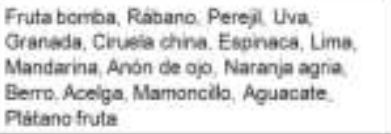 & $1-10 \%$ \\
\hline
\end{tabular}

Figura 2. Porcentaje de referencia de las plantas más utilizadas por los consumidores en su alimentación.

Leyenda: El nombre científico de las especies que aparecen en cada una de las figuras se muestra en el Anexo 1 ordenado alfabéticamente según el nombre vulgar.

En el caso de los productores (Figura 1) se puede decir de manera general que tienen una dieta balanceada con combinación de vegetales, viandas y frutas frescas. Dichos grupos fueron referidos por el $50 \%$ o más de los entrevistados, los mismos son abastecidos a partir de las producciones que obtienen de las fincas. Algo similar a lo descrito ocurre con los consumidores (Figura 2), aunque es válido destacar que entre las PDPA, que son preferidas por el $50 \%$ o más de estos, existen marcadas diferencias a pesar de algunas similitudes. Sin embargo, excepto el arroz (Oryza sativa L.) y el chícharo (Pisum sativum L.), el resto de los alimentos preferidos por los consumidores son producidos en las fincas. También se puede observar que para varias especies el porcentaje de referencia estuvo por debajo del $50 \%$.

A pesar de lo planteado, en el caso de los productores existen algunos que consumen determinados alimentos que no son producidos en sus fincas $(O$. sativa $(100 \%)$, $P$. sativum $(100$ $\%$ ), garbanzo (Cicer arietinum L.) $100 \%$ ), ñame (Dioscorea alata L.) $30 \%$ ), lechuga (Lactuca sativa L.) $20 \%$ ), zanahoria (Daucus carota L.) $20 \%$ ), pepino (Cucumis sativus L.) $20 \%$ ), col (Brassica oleracea L.) $10 \%$ ), remolacha (Beta vulgaris L.) $10 \%$ ), boniato (Ipomoea batatas (L.) Lam.) $10 \%$ ), malanga (Xanthosoma sagittifolium (L.) $10 \%$ ) y maní (Arachis hypogaea L.) 10 $\%)$. Sin embargo, el acceso a los mismos se ve garantizado al convertirse en clientes de los otros productores que aseguran la presencia de estos productos. Los porcentajes de referencia de cada uno de estos productos señalan que los más referidos por los productores entrevistados son $O$. sativa, $P$. sativum y $C$. arietinum, lo cuales adquieren a través de las redes de comercio.

Al referirse los entrevistados a la importancia de consumir y adquirir productos directamente de las fincas se pudieron distinguir tres grupos de criterios que sustentan este consumo. Todos los entrevistados le confieren importancia desde el punto de vista económico $(100 \%)$, pues adquirir productos directamente en los predios productivos disminuye los costos al ser una comercialización más directa. El resto de los criterios estuvieron relacionados con la calidad $(80 \%)$ y la inocuidad de los alimentos que adquieren $(70 \%)$. 


\section{AMAZỚNAS}

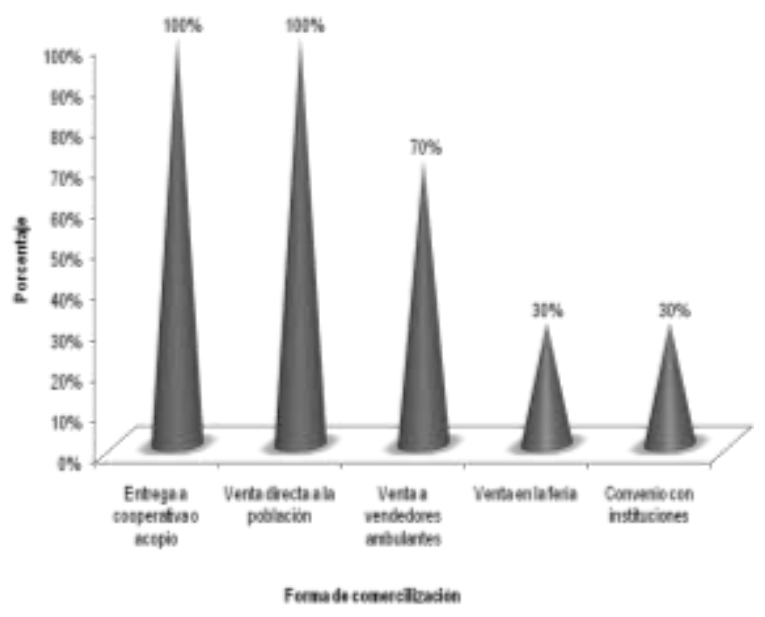

Figura 3. Formas de comercialización utilizadas por los productores.

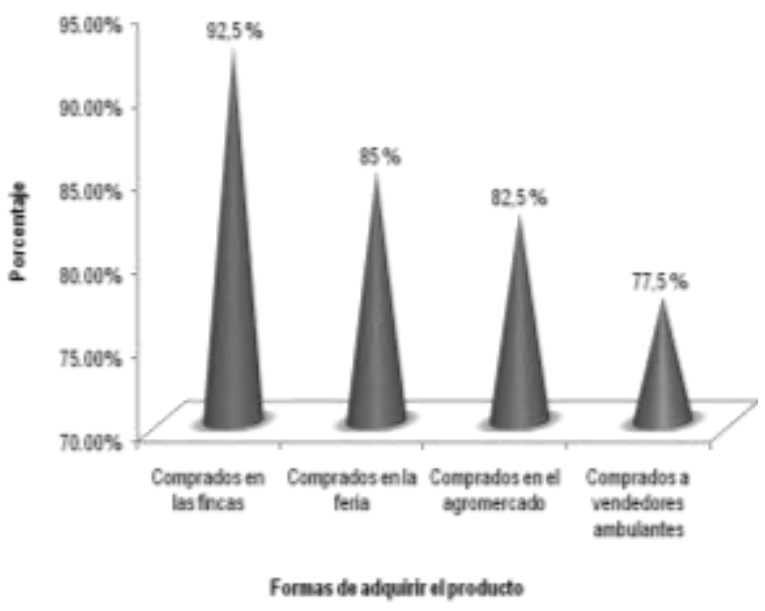

Figura 4. Vías de adquisición utilizadas por los consumidores.

Se identificaron cinco formas de comercialización utilizadas por los productores (Figura 3) donde predominaron en el $100 \%$ de los casos la entrega a la cooperativa o a acopio y la venta directa a la población. La venta a vendedores ambulantes (70 \%) y la venta en la feria o el convenio con instituciones, ambas con un $30 \%$, fueron otras formas de comercialización referidas por los principales actores en los sistemas productivos. Se pudo comprobar que todas las formas de adquisición (cinco) reportadas por los consumidores (Figura 4) guardan relación con las formas de comercialización utilizadas por los productores. Las compras en las fincas, relacionadas con las ventas directas a la población que hacen los productores, es la forma de adquisición más utilizada. También se utiliza por el $85 \%$ de los consumidores las compras en las ferias relacionadas con igual tipo de venta, las compras en el agromercado $(82,5 \%)$, que tienen su origen en la entrega que realizan los productores a las cooperativas o a acopio y finalmente la compra a vendedores ambulantes $(77,5 \%)$ relacionadas con iguales ventas por parte de los productores.

En relación con los productos que más adquieren los consumidores de acuerdo a los tipos de ventas realizadas por los productores (Tabla 1) se pueden distinguir los grupos básicos que componen la dieta alimenticia (hortalizas, viandas y frutas frescas). Lo planteado anteriormente encuentra realce si se tiene en cuenta que el $50 \%$ de los informantes señalan que realizan las compras varias veces a la semana, lo que estará condicionado por las necesidades que se presenten 
en el hogar. Se dice esto porque hay determinados productos que consumen, cuya frecuencia de compra puede ser más espaciada, lo que fue reflejado por más del $15 \%$ de los entrevistados.

\section{Tabla 1.}

Relación de productos que adquieren los consumidores según lugar de disponibilidad y tipo de venta durante el desarrollo de la investigación.

\begin{tabular}{|c|l|}
\hline $\begin{array}{c}\text { Tipo de venta } \\
\text { la finca }\end{array}$ & $\begin{array}{l}\text { Viandas: Boniato, Chopo, Malanga, Name, Plátano burro, Plátano fruta, } \\
\text { Plátano macho, Yuca. Frutas: Aguacate, Anón manteca, Cereza, Ciruela } \\
\text { china, Coco, Granada, Guayaba, Limón, Mango, Mandarina, Maracuyá, } \\
\text { Marañón, Naranja agria, Níspero, Piña, Zapote. Hortalizas: Calabaza, } \\
\text { Quimbombó, Rábano, Zanahoria. Granos y cereales: Maíz, Maní }\end{array}$ \\
\hline $\begin{array}{c}\text { Comprados en } \\
\text { el agromercado }\end{array}$ & $\begin{array}{l}\text { Viandas: Boniato, Chopo, Malanga, Name, Plátano burro. Frutas: Piña. } \\
\text { Hortalizas: Acelga, Ajo, Cebolla, Cebollín, Col, Culantro, Pepino, Perejil, } \\
\text { Pimiento, Quimbombó, Remolacha, Tomate, Zanahoria. Granos y cereales: } \\
\text { Arroz, Chícharo, Frijoles, Maíz }\end{array}$ \\
\hline $\begin{array}{c}\text { Comprados en } \\
\text { la feria }\end{array}$ & $\begin{array}{l}\text { Viandas: Chopo, Malanga, Plátano burro, Plátano macho. Frutas: Anón } \\
\text { manteca, Maracuyá, Marañón, Uva, Zapote. Hortalizas: Acelga, Ajo, }\end{array}$ \\
& $\begin{array}{l}\text { Berro, Cebolla, Culantro, Lechuga, Pepino, Pimiento, Tomate, Zanahoria. } \\
\text { Granos y cereales: Arroz, Chícharo, Frijoles, Maíz }\end{array}$ \\
\hline $\begin{array}{c}\text { Comprado a } \\
\text { vendedores } \\
\text { ambulantes }\end{array}$ & $\begin{array}{l}\text { Viandas: Boniato, Chopo, Malanga, Name, Yuca. Frutas: Coco, Guayaba, } \\
\text { Limón, Mango, Piña, Zapote. Hortalizas: Calabaza, Cebolla, Col, Lechuga, } \\
\text { Pepino, Pimiento, Tomate. Granos y cereales: Chícharo, Frijoles, Maíz }\end{array}$ \\
\hline
\end{tabular}

Leyenda: El nombre científico de las especies que se mencionan en la tabla se muestra en el Anexo 1 ordenado alfabéticamente según el nombre vulgar.

Se identificaron diferentes opciones de disponibilidad de los productos que se consumen (Figura 5). Más del $50 \%$, tanto de productores como de consumidores, consideran que los productos que utilizan como alimentos siempre están disponibles, aunque existe un grupo (el $20 \%$ de los productores y el 7,5\% de los consumidores) que encuentran algunas veces los productos que necesitan. Estos últimos señalan, además, que hay un grupo de productos que no están presentes cuando los demandan. En el caso de los productores señalaron que los productos que algunas veces no encuentran son aquellos que no son producidos en sus fincas y que tienen que comprarlos fuera del predio. De forma general el mayor acceso a los alimentos los tiene los productores.

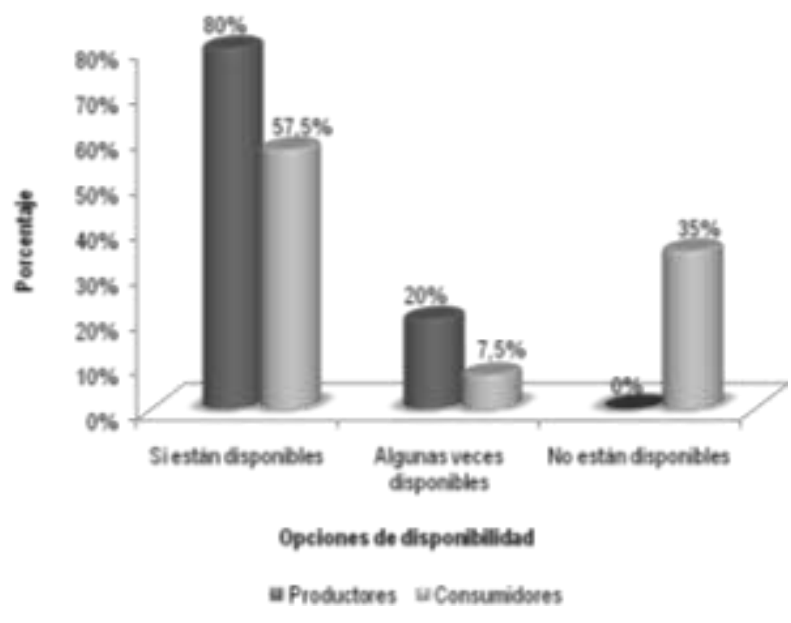

Figura 5. Disponibilidad de los productos que se consumen de según criterio de los informantes. 


\section{AMAZÖNAS}

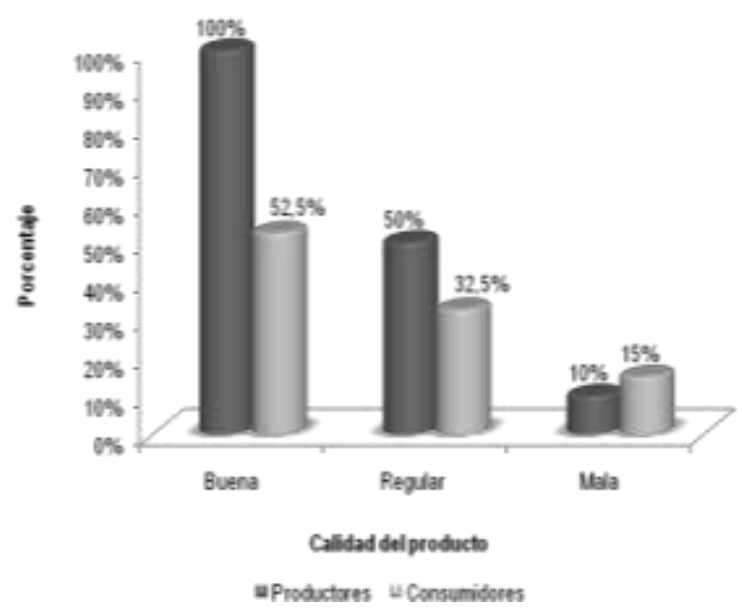

Figura 6. Calidad de los productos que se consumen según criterio de los informantes.

De acuerdo con el criterio de los informantes la calidad de los productos que son consumidos se puede clasificar en tres grupos (Figura 6). El mayor por ciento de los criterios fue para los productos que son consumidos y que tienen buena calidad, criterio que fue máximo para el caso de los productores. En el caso de los productos que tienen calidad regular hubo un porciento de referencia del $50 \%$ para el caso de los productores y del 32,5\% de los consumidores. Por su parte los productos considerados de mala calidad fueron referidos por el $10 \%$ y $15 \%$ por productores y consumidores respectivamente. Es válido destacar que según lo referido la calidad de los alimentos está asociada a los lugares donde se encuentran disponibles.

\section{Discusión de los resultados}

La composición de estos grupos de plantas tuvo un comportamiento bastante estable de un período al otro siempre con una ligera tendencia a la disminución. La variación más marcada se presentó en el total de individuos. Este indicador para el grupo de PDPA disminuyó en más de un $49 \%$ y en el caso de las EF aumentó en más del $5 \%$. Un hecho interesante es que el número de familias permanece casi sin variación entre ambos grupos de plantas. De manera general las PDPA fue el grupo que mejor composición mostró. Sin embargo, los resultados evidencian que de un período al otro puede existir cierto grado de simplificación de los predios productivos.

La existencia de numerosas especies con porcentajes de referencia por debajo del $50 \%$ demuestra que el consumo para productores y consumidores puede estar influenciado por diferentes factores. Dentro de ellos se pueden mencionar los resultados de los procesos productivos que se desarrollan en las fincas y los gustos, preferencias y condiciones de quienes producen y consumen el resultado final de la producción. De forma general los porcentajes de referencia más altos se obtuvieron para las especies mencionadas por los productores. Existe coincidencia en que plátano macho (Musa paradisiaca L.), yuca (Manihot esculenta Crantz.), tomate (Solanum lycopersicum L.), mango (Mangifera indica L.), guayaba (Psidium guajava L.), aguacate (Persea americana Mill.), I. batata, maíz (Zea mays L.) y calabaza (Cucurbita pepo L.), son los productos obtenidos de plantas que más se utilizan en la dieta, tanto de productores como de consumidores.

El que estas especies se encuentren en la preferencia ha sido reportado por otros autores. Arrieta et al. (2010), Jiménez (2011) y Pasquini et al. (2014) señalaron que las especies pertenecientes al género Musa (los plátanos) son un grupo muy frecuente y rico, además de que constituyen uno de los cultivos más importantes para la economía de la región. Otro grupo representativo es el de los mangos donde $M$. indica se encuentra reportada en la gran mayoría de los trabajos realizados 


\section{AMAZÓNAS}

sobre la APE, además es reconocida como alimento esencial dentro de la dieta (Jiménez y Estupiñán, 2011).

Otra especie con una alta frecuencia absoluta es $P$. guajava, la cual, ha sido reportada en otros trabajos como una de las especies más importantes para las comunidades, no solo como especie alimenticia sino también como medicinal. De igual forma, el cultivo de M. esculenta se reconoce como una de las principales fuentes de ingresos para los hombres en las comunidades. Asimismo, los pequeños agricultores de la región son los principales productores a nivel nacional de este alimento, gracias a que prospera en condiciones poco favorables para otros cultivos, además, su producción implica bajos costos (Alzate et al., 2010; Pasquini et al., 2014).

Dentro de los productos que son consumidos pero que no son producidos en algunas fincas objeto de estudio, D. alata, L. sativa, D. carota, C. sativus, B. oleracea, B. vulgaris, I. batatas, X. sagittifolium y A. hypogaea tuvieron porcentajes de referencia relativamente bajos. El que hayan sido referidos en menor proporción es posible que esté asociado a que su adquisición esté influenciada por el gusto de las personas que los van a consumir. Relacionado con la importancia de consumir productos obtenidos directamente de las fincas, los criterios obtenidos están sustentados sobre la base de que los productos de las fincas tienen mejor calidad y vienen de una producción mucho más orgánica y que de una forma u otra están al alcance de todos.

Por otra parte, el que exista plena coincidencia con las formas de comercialización utilizadas por los productores y las vías a partir de las cuales los consumidores adquieren los productos es importante en función de comprender la implicación de estos sistemas productivos en el logro de la SA. Lo señalado anteriormente apunta a que, por una parte, se asegura que los productos lleguen de los centros de producción a la mesa del consumidor por diferentes vías y, por otro lado, que los consumidores tengan diferentes opciones para encontrar los componentes básicos de la dieta alimentaria. Esto está asociado y a la vez tiene influencia directa en la frecuencia de compra de los diferentes productos.

En este sentido se comprobó que existan diferentes frecuencias de compras de productos para la alimentación, lo que puede variar en función del tipo de producto que se trate y de la necesidad que se presente en un momento dado. Por otra parte, estas frecuencias de compra son un reto, pues precisan de la permanencia de productos en todo momento para garantizar que los alimentos estén cuando el consumidor los necesite. Si se tienen en cuenta la relación entre los lugares de compra y las formas de consumo, se puede decir que la vegetación que se destina a la alimentación y que es producida en las fincas estudiadas, realiza una contribución importante a que las personas, diferentes a las que viven y trabajan en los predios productivos, tengan acceso a los productos para la alimentación.

Los productos que son clasificados de buena calidad son los que obtienen los productores de sus fincas y que los consumidores adquieren de manera directa. Por tanto, aquellos que ambos grupos de informantes relacionan con regular y mala calidad, son los que se adquieren por otras formas de comercialización que implican una ruta mucho más larga desde la producción hasta el consumo. De los grupos entrevistados, los productores son los que mayor acceso tienen a los productos obtenidos de las plantas y que son consumidos, esto no es contradictorio, sobre todo, si se tiene en cuenta que estas producciones realizan una contribución importante a que las personas ajenas a las fincas tengan disponibles alimentos para el consumo. De lo planteado se entiende que las fincas suburbanas objeto de estudio cumplen las funciones básicas, primero proveer de alimentos a los productores y luego con el excedente realizan acciones de comercialización.

González (2017) refirió que se estima existen 500 millones de productores agrícolas en todo el mundo que sostienen a dos mil millones de personas y representa un alto porcentaje del volumen 
de producción mundial incidiendo en los medios, la calidad de vida y la SA. A pesar que la contribución de la AF al valor sectorial parece ser inferior que el aporte que realiza la mediana y gran agricultura, este sector juega un papel crucial en el abastecimiento de alimentos básicos en la mayoría de los países de la región. En Centroamérica, la AF produce alrededor del $50 \%$ de la producción total agropecuaria de los países de la región y más del $70 \%$ de los alimentos de Centroamérica.

Otro aspecto interesante es que en muchos de los casos se pudo apreciar que los frutales de baja presencia, alimentos propios de la tradición, constituyen parte importante de la dieta tanto de los productores como de los consumidores. Los productores suburbanos fundamentalmente tienen un origen rural y al llegar al medio casi urbano los hacen con sus costumbres y tradiciones las cuales también difunden. Un comportamiento similar fue descrito por Rodríguez (2018) y Siret (2018) al estudiar sistemas de producción APE en Santiago de Cuba. Galluzi et al. (2010) señalaron que en la configuración de la APE se pueden identificar plantas con un amplio espectro de usos, que además de suplir necesidades alimentarias y constructivas, también pueden jugar un papel fundamental en las tradiciones culturales. Según Villa (2014) dentro de estas plantas se encuentran grupos introducidos frecuentemente y ricos como son los de los géneros botánicos Citrus y Dioscorea, los cuales han sido igualmente reportados como propios en la AF tropical. Agregaron además que, entre las especies nativas más frecuentes se encuentran la guanábana (Annona muricata L.), fruta bomba (Carica papaya L.) y P. americana.

De forma general se puede decir que la vegetación existente en las fincas de la agricultura suburbana contribuye entre un 55 y $67 \%$ al logro de la SA. Sin embargo, los procesos productivos que se desarrollan todavía deben centrarse un poco más en el gusto de los clientes pues hay alimentos que se producen en cantidad que no están en los principales lugares de preferencia de los consumidores. Considerar esto contribuiría a aumentar la SA y mejoraría los ingresos de los productores.

\section{Conclusiones}

Existe una composición de plantas destinadas para la alimentación bastante estable con una ligera tendencia a la disminución. Las especies vegetales sobre las cuales se sustenta la dieta de productores y consumidores tienen una representación superior al $59 \%$ en los dos períodos evaluados (poco lluvioso y lluvioso). Existe similitud en cuanto a las plantas más utilizadas por productores y consumidores para su alimentación destacándose los vegetales, las viandas y las frutas frescas. Hay correspondencia entre las formas de adquirir los productos por parte de los consumidores y las formas de comercialización utilizadas por los productores donde se destacan la entrega a la cooperativa o acopio y la venta directa a la población. Las hortalizas, viandas y frutas frescas son los productos más adquiridos por los consumidores y a los que más acceso tienen los productores con un reconocimiento superior al $50 \%$, tanto de la disponibilidad como de la calidad de los mismos. La flora cultivada con fines alimenticios en las fincas suburbanas estudiadas contribuye al logro de la seguridad alimentaria, aunque los procesos productivos todavía no satisfacen todos los gustos de los consumidores reales y potenciales.

\section{Referencias bibliográficas}

Acevedo, P. y Strong, M. (2012). Catalogue of seed plants of the West Indies. Washington: Smithsonian Institution.

Alzate, G., Vallejo, F. A., Ceballos, H., Pérez, J. y Fregene, M. (2010). Variabilidad genética de la yuca cultivada por pequeños agricultores de la región Caribe de Colombia. Acta Agronómica, 20(2), 309-328.

Ardón, C. R. (2012). La formación de recursos humanos como estrategia de apoyo a la seguridad alimentaria y nutricional (SAN). El caso de la educción superior en Honduras. (Tesis 


\section{AMAZÚNAS}

presentada en opción al título de Doctor en Ciencias). Universidad Politécnica de Madrid, España, Madrid.

Arrieta, R., García, A. y Leviller, L. (2010). Hombre y desnutrición en Bolívar: un análisis desde el enfoque de la equidad y la seguridad alimentaria. Cartagena: Unicartegena.

Castro, S. (2013). Introducción. En M. d. Defensa, Seguridad alimentaria y seguridad global (págs. 11-23). Madrid-España: Ministerio de Defensa.

Consejo Nacional de Evaluación de la Política de Desarrollo Social [CONEVAL]. (2010). Dimensiones de la seguridad alimentaria: evaluación estratégica de nutrición y abasto. México, D.F.: CONEVAL.

del Toro, J. O., Vargas, B., Rizo, M. y Candó, L. (2018). Composición, estructura y distribución de la vegetación arvense existente en fincas de la agricultura suburbana en Santiago de Cuba. Revista Científica Agroecosistemas, 6(1), 68-81. Recuperado de https://aes.ucf.edu.cu/index.php/aes/article/view/166/201.

del Toro, J. O., Vargas, B., Rodríguez, R., Rodríguez, E. J., Fernández, D. y Ramos, Y. M. (2019). Potencialidades de la vegetación arvense existente en fincas de la agricultura suburbana de Santiago de Cuba para la alimentación. Agrisost, 25(2), 1-5. Recuperado de https://revistas.reduc.edu.cu/index.php/agrisost/article/view/e2747.

Galluzi, G., Eyzaguirre, P. y Negri, V. (2010). Home gardens: neglected hotspots of agrobiodiversity and cultural diversity. Biodiversity and Conservation, 19(13), 3635-3654.

González, R. (2017). Contribución de la flora existente en fincas suburbanas de Santiago de Cuba al logro de la seguridad alimentaria. (Tesis presentada en opción al título de Ingeniero Agrónomo). Universidad de Oriente, Santiago de Cuba, Cuba.

Greuter, W. y Rankin, R. (2017). Plantas vasculares de Cuba. Inventario preliminar. Berlín: Botanischer Museum Berlín-Universidad de La Habana.

Guardiola, J., González, V. y Vivero, J. L. (2006). La seguridad alimentaria: estimación de índices de vulnerabilidad en Guatemala. VIII Reunión de Economía Mundial. Alicante: CEUETEA.

Guerrero, D. (2017). Diversidad vegetal en fincas de la agricultura suburbana enSantiago de Cuba: sus potencialidades para el control natural de plagas. (Tesis presentada en opción al título de Ingeniero Agrónomo). Universidad de Oriente, Cuba, Santiago de Cuba.

High Level Panel of Experts [HLPE]. (2013). Inversión en la agricultura a pequeña escala en favor de la seguridad alimentaria. Roma: HLPE

Instituto de Nutrición de Centro América y Panamá [INCAP]. (2004). La seguridad alimentaria y nutricional: un enfoque integral. Guatemala: INCAP.

Jiménez, N. (2011). Huertos familiares en la bahía de Cispatá. Bonplandia, 20(2), 309-328.

Jiménez, N. D. y Estupiñán, A. (2011). Useful Trees of the Caribbean Region of Colombia. Bioremediation, Biodiversity and Bioavailability, 5(Special Issue 1), 65-79.

Lipper, N. y Neves, L. (2011). Los intercambios campesinos: más allá de las fronteras. San José, Costa Rica: IICA-GTZ-CIRAD.

Ministerio de la Agricultura [MINAG]. (2015). Lineamientos de la Agricultura Urbana, Suburbana y Familiar. La Habana: MINAG-INIFAT-ACTAF.

Orellana, R. (2010). A propósito de un concepto: Suburbanización. Agricultura Orgánica, (3), 31-32.

Organización de la Naciones Unidas para la Alimentación y Agricultura [FAO]. (16 de noviembre de 2010). Perspectivas de alimentación-Analyse des marches mondiaux. Seguridad alimentaria: - definiciones y causales, 1-28.

Organización de la Naciones Unidas para la Alimentación y Agricultura [FAO]. (2011b). Una introducción a los conceptos básicos de la seguridad alimentaria. Roma-Italia: FAO.

Organización de la Naciones Unidas para la Alimentación y Agricultura [FAO]. (2011a). Ahorrar para crecer, capítulo 1. Recuperado el 10 de junio de 2012, de http://www.fao.org

Organización de las Naciones Unidas para la Alimentación y la Agricultura [FAO]. (2015). Biodiversidad y servicios ecosistémicos. Italia, Roma: FAO. 
Páez, E. (2013). La agricultura urbana y suburbana en el contexto cubano. Agricultura Orgánica, 19(1), 15-17.

Pasquini, M., Sánchez, C. y Mendosa, J. (2014). Distribución del conocimiento y usos por generación y género de plantas comestibles en tres comunidades afrodescendientes en Bolívar. Luna Azul, 38, 58-85.

Proctor, F. y Lucchesi, V. (2012). La agricultura a pequeña escala y en los mercados de productos agroalimentarios. London-The Hague-La Paz: IIED-Hivos-Mainumby.

Rah, J. H., Akhter, N., Semba, R. D., de Pee, S., Bloem, M. W., Campbell, A. A., y otros. (2010). Low dietary diversity is a predictor of child stunting in rural Bangladesh. European Journal of Clinical Nutrition, (64), 1393-1398.

Rodríguez, E. J. (2018). Comportamiento de especies vegetales y criterios sociales que sustentan su presencia en la agricultura familiar en Santiago de Cuba. (Tesis presentada en opción al título de Ingeniero Agrónomo). Universidad de Oriente, Santiago de Cuba, Cuba.

Roig, J. T. (1988). Diccionario Botánico de Nombres Vulgares Cubanos. La Habana: CientíficoTécnica.

Secretaria del Convenio sobre la Diversidad Biológica [CDB]. (2008). La Biodiversidad y la Agricultura: Salvaguardando la biodiversidad y asegurando alimentación para el mundo. Montreal, Canadá: CDB.

Siret. A. (2018). Diversidad vegetal en patios familiares y su aporte a la seguridad alimentaria en Santiago de Cuba. (Tesis presentada en opción al título de Ingeniero Agrónomo). Universidad de Oriente, Santiago de Cuba, Cuba.

Valdés, H. (2017). Potencialidades de tres grupos de plantas en agroecosistemas suburbanos para generar bienes y servicios en Santiago de Cuba. (Tesis presentada en opción al título de Ingeniero Agrónomo). Universidad de Oriente, Santiago de Cuba, Cuba,

Vargas, B. (2006). Efecto del extracto crudo de Wedelia trilobata y Tagetes erecta en el control de Alternaria solani y Alternaria porri en condiciones in vitro. (Tesis presentada en opción al título de Ingeniero Agrónomo). Universidad de Granma, Granma, Cuba.

Vargas, B., Candó, L., Pupo, Y. G., Ramírez, M., Escobar, Y., Rizo, M., Molina, L. B., Bell, T. D. y Vuelta, D. R. (2016). Diversidad de especies vegetales en fincas de la agricultura suburbana en Santiago de Cuba. Agrisost, 22(2), 1-23. Recuperado de http://www.agrisost.reduc. edu.cu.

Vargas, B., Candó, L., Ramírez, M., Rizo, M., Pupo, Y. G., González, L., Vuelta, D. R., Bell, T. D. y Molina, L. B. (2017). Diversidad de plantas objeto de cultivo en cuatro fincas de la agricultura suburbana de Santiago de Cuba. Agrisost, 23(3), 90-110. Recuperado de: http://www.agrisost.reduc.edu.cu.

Vargas, B., del Toro, J. O., Pupo, Y. G., Rizo, M., Candó, L., Ferrer, J. C. (2020) Percepción etnobotánica de los campesinos sobre la flora arvense en fincas de la agricultura suburbana en Santiago de Cuba, Cuba. Boletín Latinoamericano y del Caribe de Plantas Medicinales y Aromáticas, 19(1): 126-141. Doi: https://doi.org/ 10.37360/blacpma.20.19.1.6

Vargas, B., del Toro, J. O., Rodríguez, E. J., Rizo, M. y Pupo, Y. G. (2019b). Potencialidades medicinales de la flora arvense en fincas suburbanas de Santiago de Cuba. Centro Agrícola, 46(1), 54-57. Recuperado de http://cagricola.uclv.edu.cu.

Vargas, B., González, R., Rodríguez, R. y Garcés, W. (2019a). Composición, diversidad y distribución de especies frutales en fincas suburbanas de Santiago de Cuba. Universidad y Sociedad, 11(3), 94-105. Recuperado de http://rus.ucf.edu.cu/index. php/rus.

Vargas, B., Pupo, Y., Puertas, A., Mercado, I. y Hernández, W. (2011). Estudio etnobotánico sobre tres especies arvenses en localidades de la región oriental de Cuba. Granma Ciencia, $15(3)$.

Vázquez, L. L. (2017). Manejo de plagas en la agricultura urbana y suburbana. Síntesis de la experiencia en Cuba. III Congreso Internacional de Agricultura Urbana, Suburbana y Familiar (pág. 80). La Habana: INIFAT. 
Velasco, D. (2014). Modelo de comercialización del Nopal orgánico en la Delegación Milpa Alta. (Tesis para obtener el grado de Maestro en Administración). Instituto Politécnico Nacional, Distrito Federal, México.

Villa, D. L. (2014). Composición y Riqueza de las Plantas Alimenticias En Huertos Familiares De San Pablo. Bogotá: Pontifica Universidad Javeriana, Colombia.

\section{Anexo 1.}

Listado del nombre científico de las especies mencionadas en las investigaciones organizadas alfabéticamente a partir del nombre vulgar.

Acelga (Beta vulgaris var. Cicla L.) Aguacate (Persea americana Mill.) Ajo (Allium sativum L.)

Anón de ojo (Annona squamosa L.)

Anón manteco (Annona reticulata L.)

Arroz (Oryza sativa L.)

Berenjena (Solanum melongena L.)

Berro (Nasturtium officinale W.T. Aiton)

Boniato (Ipomoea batatas (L.) Lam.)

Café (Coffea arabica L.)

Calabaza (Cucurbita pepo L.)

Cañandonga (Cassia grandis L.)

Cebolla (Allium cepa L.)

Cebolla multiplicadora (Allium cepa var.

Aggregatum Dom.)

Cebollín (Allium canadense L.)

Cereza (Malpighia glabra L.)

Ciruela china (Averrhoa carambola L.)

Coco (Cocos nucifera L.)

Col (Brassica oleracea L.)

Culantro (Eryngium foetidum L.)

Chícharo (Pisum sativum L.)

Chopo (Colocasia esculenta (L.) Schott)

Espinaca (Spinacia oleracea L.)

Frijoles (Phaseolus spp.)

Frijol caballero (Phaseolus lunatus L.)

Frijol gandul (Cajanus cajan (L.) Huth.)

Fruta bomba (Carica papaya L.)

Granada (Punica granatum L.)

Guanábana (Annona muricata L.)

Guayaba (Psidium guajava L.)

Habichuela (Vigna unguiculata (L.) Walp.)

Lechuga (Lactuca sativa L.)

Lima (Citrus limetta Risso.)
Limón (Citrus limon (L.) Osbeck.)

Maíz (Zea mays L.)

Malanga (Xanthosoma sagittifolium (L.)

Schott.)

Mamoncillo (Melicoccus bijugatus Jacq.)

Mandarina (Citrus reticulata Blanco.)

Mango (Mangifera indica L.)

Maní (Arachis hypogaea L.)

Maracuyá (Passiflora edulis Sins.)

Marañón (Anacardium occidentales L.)

Melón (Cucumis melo L.)

Naranja agria (Citrus aurantium L.)

Níspero (Manilkara zapota (L.) P. Royen)

Name (Dioscorea alata L.)

Pepino (Cucumis sativus L.)

Perejil (Petroselinum crispum (Mill.)

Nymam ex. A. w. Hill.)

Pimiento (Capsicum annuum L.)

Piña (Ananas comosus (L.) Merr.)

Plátano burro (Musa sp.)

Plátano fruta (Musa sapientum L.)

Plátano macho (Musa paradisiaca $\mathrm{L}$.)

Quimbombó (Abelmoschus esculentus (L.)

Moench.)

Rábano (Raphanus sativus L.)

Remolacha (Beta vulgaris L.)

Sorgo (Sorghum bicolor (L.) Moench.)

Tomate (Solanum lycopersicum L.)

Uva (Vitis vinifera L.)

Yuca (Manihot esculenta Crantz.)

Zanahoria (Daucus carota L.)

Zapote (Pouteria sapota (Jacq.) H.E. Moore $\&$ Stearn.) 